\title{
Swimmers Heal on the Move Following
}

\section{Catastrophic Damage}

Emil Karshalev ${ }^{\mathrm{\dagger}}$, Cristian Silva-Lopez ${ }^{\mathrm{a}} \uparrow, \mathrm{Kyl}_{\mathrm{e}}$ Chan $^{\mathrm{b}}$, Jieming Yan ${ }^{\mathrm{a}}$, Elodie Sandraz ${ }^{\mathrm{a}}$, Mathieu Gallot ${ }^{\mathrm{a}}$, Amir Nourhani ${ }^{\mathrm{a}}$, Javier Garay ${ }^{\mathrm{b}}$ and Joseph Wang ${ }^{\text {a* }}$

a. Department of NanoEngineering, University of California San Diego, La Jolla, CA 92093, USA.

b. Department of Mechanical and Aerospace Engineering, University of California San Diego, La Jolla, CA 92093, USA.

$\dagger$ E.K. and C. S.-L. contributed equally to this work.

*Corresponding Author email: josephwang@eng.ucsd.edu

\section{MATERIALS AND METHODS}

Fabrication of SHSs: The fabrication process was comprised of printing various functional inks. The components of the swimmer body and tail were designed in AutoCAD (Autodesk, San Rafael, CA) and used as patterns in the $12^{\prime \prime} \times 12^{\prime \prime}$ stainless steel stencils (Metal Etch Services, San Marcos, CA). A temporary tattoo paper sheet (Papilio, HPS LLC, Rhome, TX), precoated with a water-soluble adhesive, served as the substrate for the printing process. A typical SHS included the printing of three layers using an MPM SPM semi-automatic screen printer (Speed-line Technologies). First, the conductive layer from graphite ink (E3449, Ercon, Inc., Wareham, MA) was printed 
in the shape of the swimmer and cured at $60{ }^{\circ} \mathrm{C}$ in an oven. This conductive layer enabled subsequent electrodeposition of Pt on the tail to enable propulsion. Next, three consecutive layers of a hydrophobic, rigid layer was printed. The hydrophobic layer consists of $30 \mathrm{wt} \%$ solution of polystyrene-polymethylmethacrylate copolymer (Aldrich, St. Louis, MO) in toluene with $5 \mathrm{wt} \%$ aerogel particles $(<20 \mu \mathrm{m}$, Jios Chemicals, South Korea). Lastly, magnetic $\mathrm{Nd}_{2} \mathrm{Fe}_{14} \mathrm{~B}$ powder was obtained following previously reported protocols. ${ }^{[1]}$ Fabrication of the magnetic ink consisted of preparing a $30 \mathrm{wt} \%$ dispersion of magnetic powder in polymeric insulator ink (Dupont 5036, Dupont, Wilmington, DE). In order to align the magnetic particles, the SHS was exposed to a strong magnetic field using a commercial magnet (CMS Magnetics).

Deposition of Catalytic Pt: Swimmers were removed from the tattoo paper substrates by soaking them in water for $30 \mathrm{~s}$ and then sliding the SHS off the edge of the substrate. Next, SHSs were subjected to an electrochemical deposition of a Pt layer on their tail using a commercial Pt plating solution (Technic Inc., Anaheim, CA) in a standard threeelectrode setup. The carbon tail of the swimmer served as the working electrode, while a Pt wire and $\mathrm{Ag} / \mathrm{AgCl}(1 \mathrm{M} \mathrm{KCl})$ served as the counter and reference electrodes, 
respectively. The galvanostatic deposition of $\mathrm{Pt}$ was performed on a $\mu$-Autolab potentiostat (Metrohm Autolab B. V., Netherlands) at $-2 \mathrm{~mA}$ for 20,60 or $120 \mathrm{~s}$.

Swimming and Self-Healing: Self-healing swimmers were tested inside a $150 \mathrm{~mm}$ diameter Petri dish filled with $70 \mathrm{~mL}$ of $15 \% \mathrm{H}_{2} \mathrm{O}_{2}$ solution. A camera mounted on a tripod was placed above the Petri dish to record videos of the swimming and healing processes. Damage to the SHSs was introduced with a blade and the SHS pieces were placed back into the solution in opposite sides of the Petri dish and left to autonomously heal.

Magnetic Hysteresis and Field Distribution Measurement: A Quantum Design Versalab with a VSM attachment was used to measure the magnetic hysteresis of the SHSs. Printed magnetic strips were measured at room temperature (300K) with an applied field sweeping from -30 to $30 \mathrm{kOe}$. Each strip was measured in two orientations which were perpendicular to each other (hereafter designated as D1 and D2). The direction D1 denotes the orientation perpendicular to the magnetic alignment inside the strip while D2 denotes the orientation parallel to the magnetic alignment in the strip. 
Measurements of the actual field distribution around an SHS were performed with a Lakeshore Model 425 Gauss meter and probe. An area of $35 \times 40 \mathrm{~mm}$ was separated into a $7 \times 8$ grid with an individual box size of $5 \times 5 \mathrm{~mm}$. The SHS was placed in the middle of the grid and at each grid box the value of the magnetic field was measured to produce a field distribution map.

Equipment: Videos were taken on a Nikon D7000 camera with a Micro Nikon 40 mm lens mounted on a tripod. The resulting videos were analyzed with Nikon Elements AR 3.2 tracking software.

Statistical Analysis: All quantitative values were presented as means \pm SD. All experiments (each model, Pt deposition scenario and cut position) were performed for at least three independent repeats. Healing efficiencies were calculated from 3 healing events per each independent model or cut position.

\section{REFERENCES}

1. Bandodkar, A. J.; López, C. S.; Mohan, A. M. V.; Yin, L.; Kumar, R.; Wang, J. AllPrinted Magnetically Self-Healing Electrochemical Devices. Sci. Adv. 2016, 2, e1601465. 

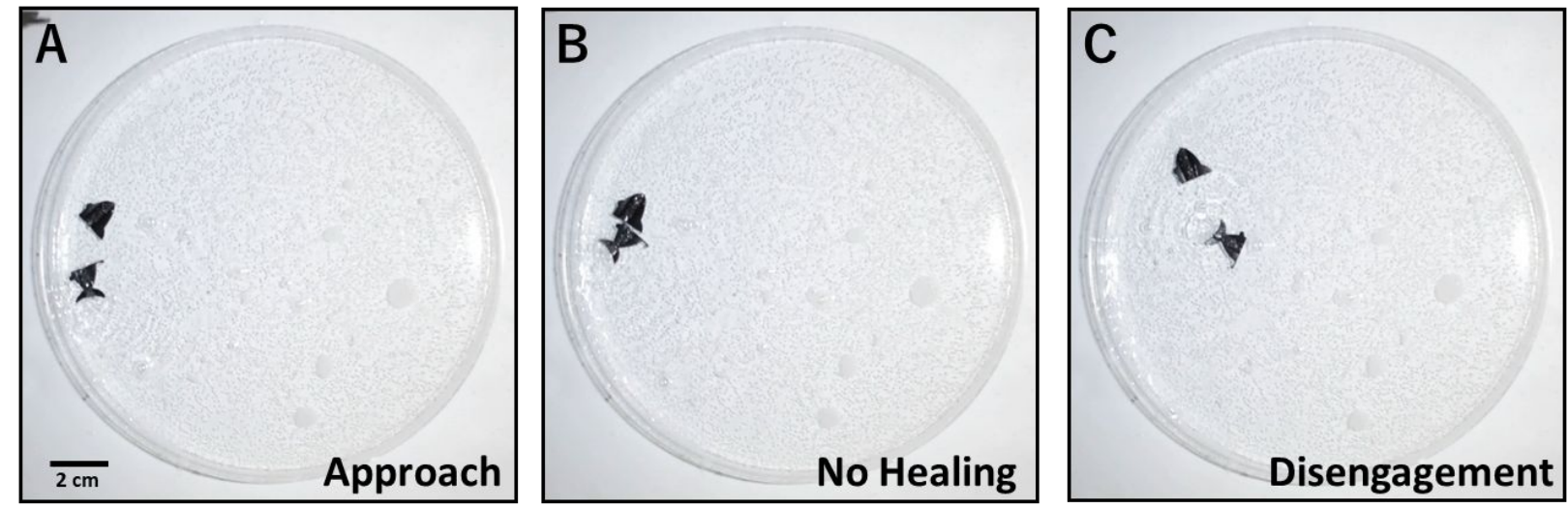

Figure S1. Unsuccessful healing of a model 1U SHS. (A) Two parts of the swimmer approach each other. (B) The pieces touch but no reattachment occurs. (C) The self-propelling piece moves away from the static piece.

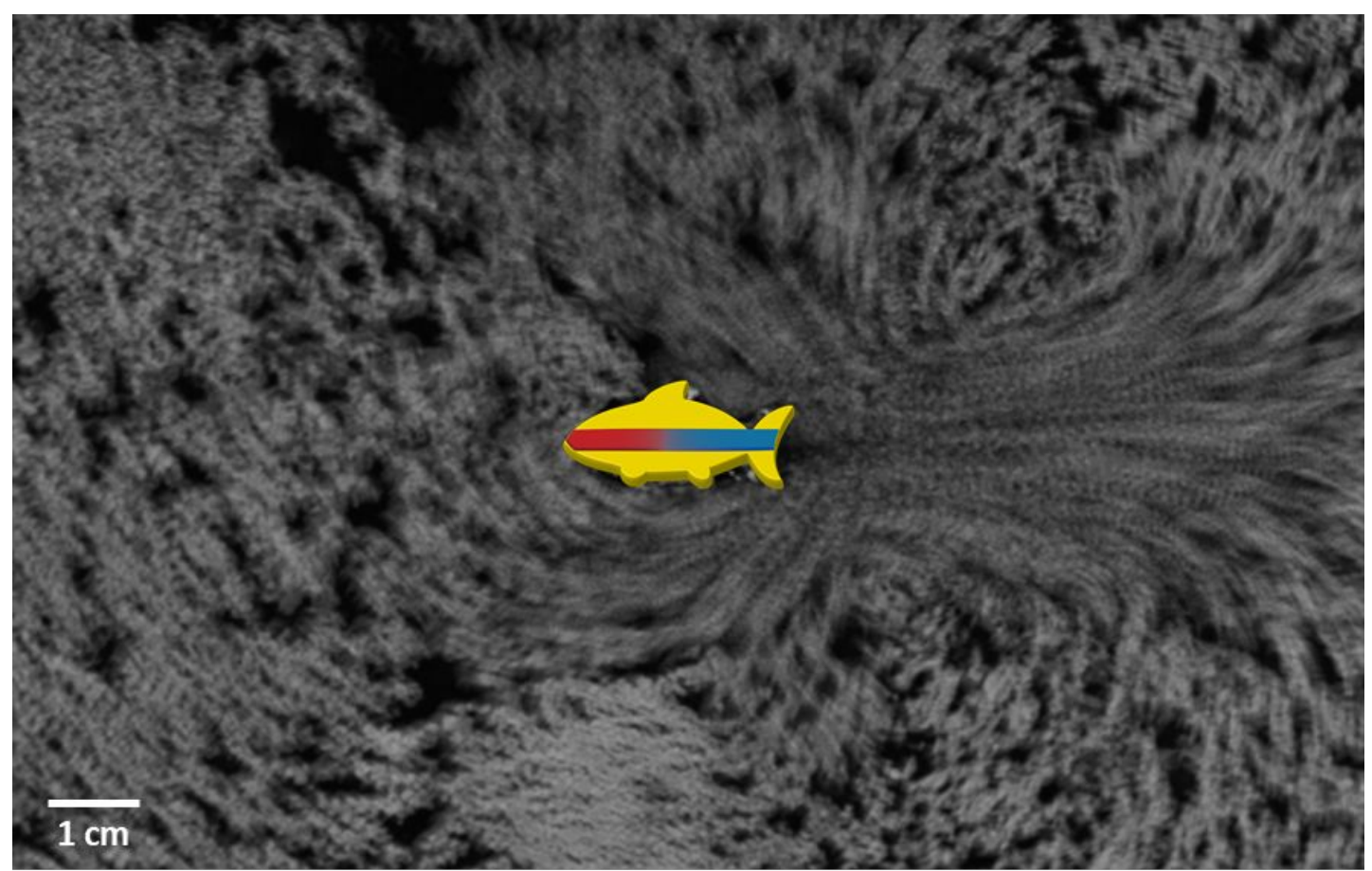

Figure S2. Flow pattern around a SHS. 
To consider the role of fluid dynamics upon the efficiency of such 'on-the-fly' healing, an experiment where a swimmer was immobilized (fixed) to the surface but allowed to react with the fuel and produce bubble thrust was performed. Tracer particles were introduced to capture the flow of the liquid around the confined swimmer. For a typical SHS of length scale $\sim 1 \mathrm{~cm}$ moving with speed of $\sim 10 \mathrm{~cm} / \mathrm{s}$ in a fluid of density $\sim 1000$ $\mathrm{kg} / \mathrm{m}^{3}$ and viscosity $\sim 0.001$ Pa.s the Reynolds number is about 1000 . Far from the low Reynolds number regime inertia can have a significant influence on swimmer motion. Figure S2 shows the flow field around a fixed swimmer. The flow pattern near the tail differs significantly from the flow pattern in a steady flow (around the rest of the swimmer). The bubbles leave the tail and apply momentum to the fluid. Upon bursting on the surface, the bubbles push the fluid away from the tail and provide the propulsion force. Considering this fluid dynamic behavior, we expect self-healing to be dictated not only by the magnetic attraction but also by the dynamics of the propulsion.

To gain a better understanding of the magnetic forces and how they vary with distance and magnetic strip separation we developed an analytical model (corresponds to Fig 3E). The separated strips behave as two permanent magnets with lengths $\mathrm{L}_{1}$ and $\mathrm{L}_{2}$ and $\mathrm{L}=\mathrm{L}_{1}+\mathrm{L}_{2}$. We define the fraction $\mathrm{x}=\mathrm{L}_{1} / \mathrm{L}$ as the fraction of the strip that contains all or part of the tail. A 
magnetic strip on a self-healing swimmer has a typical length $L=20 \mathrm{~mm}$, a width of $1=2.5 \mathrm{~mm}$ and thickness of $t=0.270 \mathrm{~mm}$. Using the width as a length scale, $t / 1 \sim \Theta\left(10^{-1}\right)$ we can approximate the magnetic bars as two-dimensional objects. Using the method of magnetic pole strength and approximating the bars as 2D rectangles we obtain the magnetic force between the strips (Equation 1)

$$
F_{m}\left(L_{1}, L_{2}\right)=f\left(L_{1}+d\right)+f\left(L_{2}+d\right)-f(L+d)-f(d)
$$

$$
\text { with } f(x)=\frac{\mu_{0}}{2 \pi}(t M)^{2}\left[\sqrt{1+\left({ }^{l} / x^{2}\right.}-1\right] \text { where } \mu_{0}=4 \pi \times 10^{-7} \mathrm{H} / \mathrm{m} \text { is the vacuum permeability, }
$$

$\mathrm{t}$ is the thickness of the magnetic strip, 1 is the width of the magnetic strip, and $\mathrm{M}$ is the magnetization density. Defining the force scale as $F^{*}=\left[\frac{\mu_{0}}{2 \pi}(t M)^{2}\right]$, the dimensionless force between the bars $\tilde{F}_{m}=F_{m} / F^{*}$ is plotted vs the dimensionless distance $(d / l)$ for different cut positions ( $\mathrm{x}$ ) and an aspect ratios of the magnetic strips $\mathrm{L} / \mathrm{l}=8$ as it corresponds to a Model 1 swimmer (where $\mathrm{d}$ is the separation distance between the magnetic strips). As the plots shows, the maximum attraction force happens when the magnetic strip parts into two equal segments $(\mathrm{x}=0.5)$. The force between the magnetic strips drops rapidly as a function of distance between the nearest poles. Thus, one of the main roles of magnetic attraction in the self-healing process is the alignment of the orientation of the magnetic strips to facilitate 'on-the-fly' healing. 

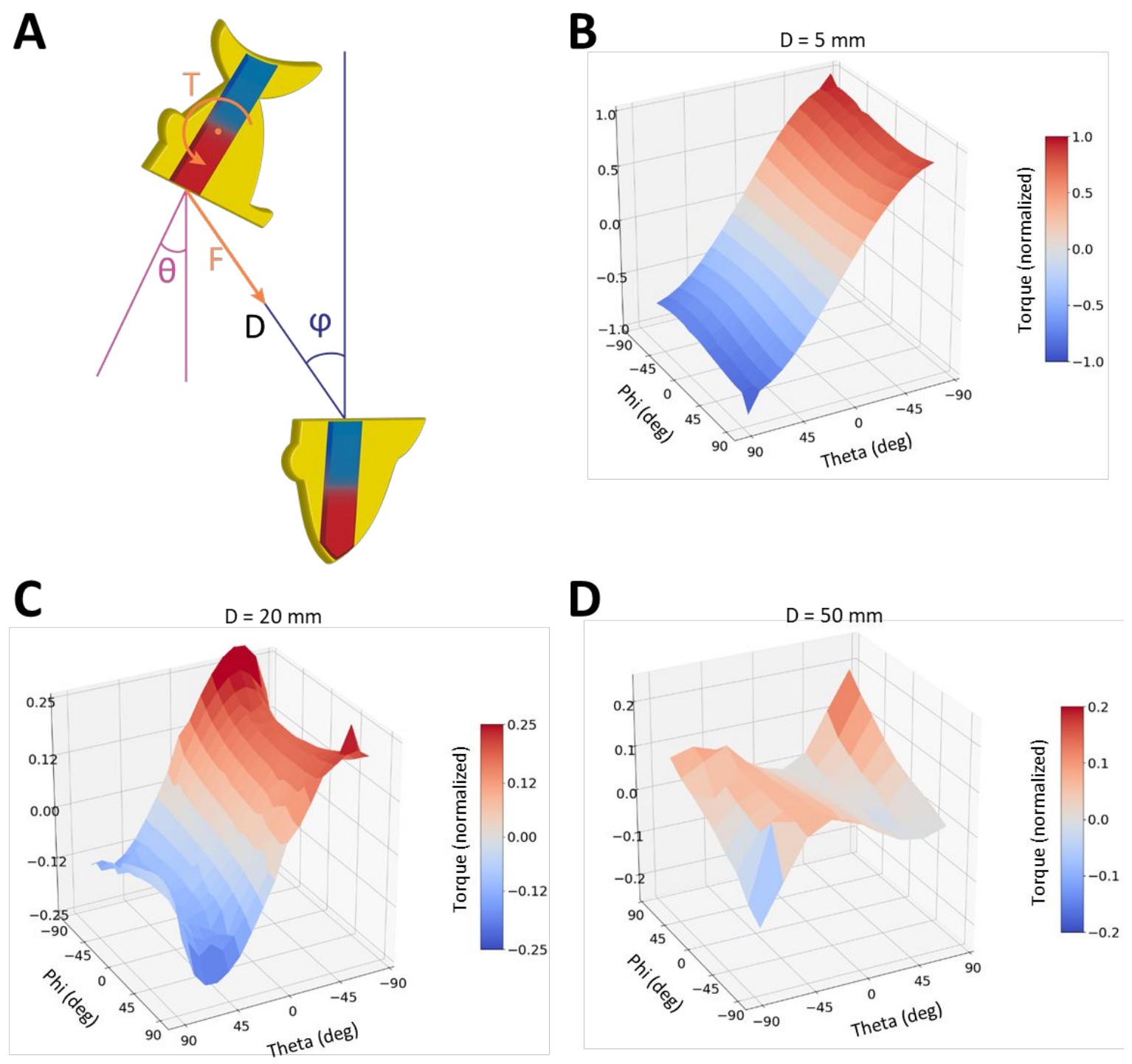

Figure S3. Simulation of the magnetic torque towards alignment in different scenarios.

(A) Schematic representation of the orientations a damaged swimmer can assume depending on the rotation angle $(\theta)$, approach angle $(\varphi)$, and distance (D). Normalized torque as a function of the rotation and approach angles for small (B), medium (C), and large (D) distances.

COMSOL Multiphysics 5.2 software with the AC/DC $>$ Magnetic Fields, no Currents (mfnc) module was chosen along with a two-dimensional Stationary Study. The study was focusing on the magnetic interactions between the two magnetic strips comprising the swimmers in order to deduce trends of the magnetic forces and of magnetic torques for various positions of the stripes (varying distance and orientation between them). The two magnetic strips were modeled as 2 identical rectangles made out of $\mathrm{Nd}_{2} \mathrm{Fe}_{14} \mathrm{~B}$, in a surrounding disk of air. The first strip had 
a fixed position in the center of the geometry while the latter one was positioned in a half-disk around the first one where their poles could face each other Figure S3A. Precaution should be taken using COMSOL for such model and one should consider the effects of boundary conditions, the appearance of numerical noise and the impact of the meshing. The dimensions of the circle of air were set to 100 times larger than the length of the stipes to prevent boundary conditions from affecting the magnetic field. The meshing has been tuned to be extremely fine on the stripes, fine in a 2-meters circle surrounding the swimmers and coarse over 2 meters. Finally, as the theory assures that the magnetic forces applied from one magnet to another are equal, we computed the numerical noise as the relative difference between the two forces and tuned the meshing to make sure that this noise would not exceed $5 \%$ in the range of the positions we used. The relative magnetic permeability of the stripes has been set to 1.05 and the magnetization to $468 \mathrm{kA} / \mathrm{m}$ (Figure $2 \mathrm{~B}$ ) along their length. The magnetic torque has been computed on the center of mass of the moving strip. Positions have been chosen to describe the behavior of the mobile magnet from $-80^{\circ}$ to $+80^{\circ}$ around the immobilized magnet $(\varphi)$, from $80^{\circ}$ to $+80^{\circ}$ around its center of mass $(\theta)$ and the distance between them from $5 \mathrm{~mm}$ to $50 \mathrm{~mm}$.

Here, we refrained from adding the dynamic motion of the tail sections to remove temporal changes and elucidate the aligning nature of the magnetic interaction (Figure S3). We chose to look at torque because this quantity is directly responsible for the rotation and alignment of magnetic strips at various orientations. At small distances (5 $\mathrm{mm}$ ) the torque increases as the rotation of the mobile strip grows more perpendicular to the stationary one. This is expected as the more misaligned (perpendicular) the 
strips are the larger the torque will be to bring them back to alignment. However, upon increasing the distance between the strips $(20$ and $50 \mathrm{~mm}$ ) the torque assumes a maximum before the perpendicular case, suggesting that the alignment is preferred at smaller rotation angles $(\theta)$. Additionally, when the approach angle is large $(\varphi)$ the torque is higher for virtually all cases, suggesting that the susceptibility for alignment is stronger on the periphery of the half-disk described by $\varphi$. Conceptually, it makes sense that the torque is low at low angles of $\varphi$ and $\theta$ as the strips are already nearly aligned, suggesting high probability of healing. With more misalignment the torque is higher, meaning an induced rotation of the swimmer will occur to align them and produce recombination. Finally, at large values of $\theta, \varphi$ and $D$ the torque will surpass a maximum and decrease, suggesting that beyond these points self-healing is more unlikely. Overall, the data of Figure S3 suggest that the magnetic torque will most likely align damaged pieces at all orientations at short distances while at large distances the orientations which will lead to healing become more restricted. 

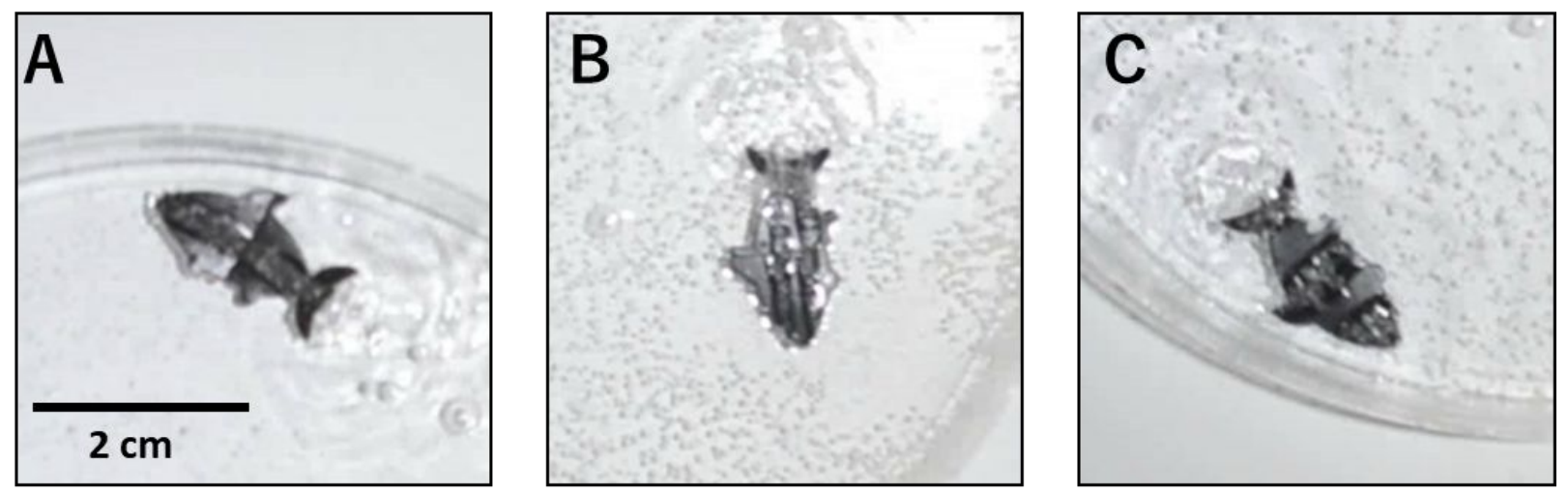

Figure S4. Zoom-ins of self-healed model 1 (A), model 2 (B) and model 3 (C) swimmers, respectively, from Figure 4 .
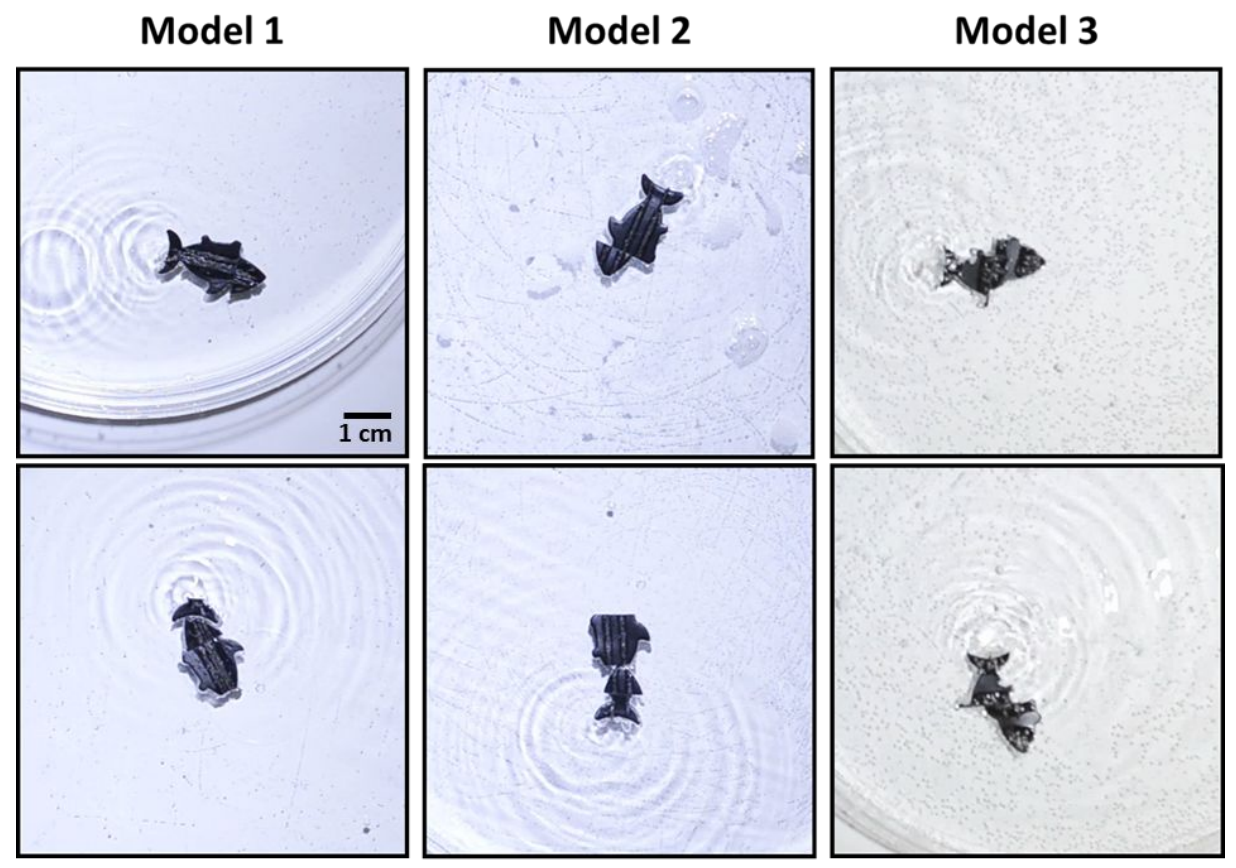

Figure S5. Misaligned healing. Examples of misaligned healing of model 1, 2 and 3 SHSs featuring misalignment of the magnetic strips and incorrect order of healing for the 2 cut cases.

Video S1. Self-Healing Swimmers

Video S2. Magnetic Field Distribution During Self-Healing

Video S3. Unsuccessful Healing of Model 1U Swimmer

Video S4. Effect of Cut Position on Self-Healing

Video S5. Effect of Strip Geometry on Self-Healing 
